ARTICLE OPEN

\title{
Enhancing the brightness of electrically driven single-photon sources using color centers in silicon carbide
}

\author{
Igor A. Khramtsov ${ }^{1}$, Andrey A. Vyshnevyy (D) ${ }^{1}$ and Dmitry Yu. Fedyanin (iD)
}

Practical applications of quantum information technologies exploiting the quantum nature of light require efficient and bright true single-photon sources which operate under ambient conditions. Currently, point defects in the crystal lattice of diamond known as color centers have taken the lead in the race for the most promising quantum system for practical non-classical light sources. This work is focused on a different quantum optoelectronic material, namely a color center in silicon carbide, and reveals the physics behind the process of single-photon emission from color centers in SiC under electrical pumping. We show that color centers in silicon carbide can be far superior to any other quantum light emitter under electrical control at room temperature. Using a comprehensive theoretical approach and rigorous numerical simulations, we demonstrate that at room temperature, the photon emission rate from a $\mathrm{p}-\mathrm{i}-\mathrm{n}$ silicon carbide single-photon emitting diode can exceed 5 Gcounts/s, which is higher than what can be achieved with electrically driven color centers in diamond or epitaxial quantum dots. These findings lay the foundation for the development of practical photonic quantum devices which can be produced in a well-developed CMOS compatible process flow.

npj Quantum Information (2018)4:15; doi:10.1038/s41534-018-0066-2

\section{INTRODUCTION}

Silicon carbide has been a recognized material for high-power and high-temperature electronics for several decades. ${ }^{1}$ At the same time, despite the fact that light emission from semiconductors was for the first time observed from silicon carbide ${ }^{2-4}$ and $6 \mathrm{H}-\mathrm{SiC}$ yellow light emitting diodes were serially produced in the USSR in the 1970s, for a long time SiC could not find applications in the optoelectronics of the XXI century. Due to the indirect bandgap and thus low efficiency of light-matter interaction, SiC could not compete with other semiconductor materials. However, recently it attracted great research interest owing to the progress in an entirely different field of research.

After the first studies of color centers in diamond, ${ }^{5-7}$ it has become clear that point defects in the crystal lattice of dielectrics and wide-bandgap semiconductors can be efficiently used in quantum information technologies. These defects can be created in diverse solid-state structures. At the same time, their optical properties are much closer to the properties of isolated atoms and molecules than those of quantum dots. ${ }^{8}$ This gives a unique opportunity to exploit the quantum optics effects at room temperature. ${ }^{9}$

Silicon carbide, as well as diamond, can host diverse color centers, such as carbon antisite-carbon vacancy complexes $\left(\mathrm{C}_{\mathrm{Si}} \mathrm{V}_{\mathrm{C}}\right)_{1}{ }^{10-12}$ divacancies $\left(\mathrm{V}_{\mathrm{C}} \mathrm{V}_{\mathrm{Si}}\right)_{1,18}^{13,14}$ silicon vacancies $\left(\mathrm{V}_{\mathrm{Si}}\right)_{1}^{12,15,16}$ nitrogen-vacancy (NV) centers, ${ }^{17,18}$ silicon antisite-stacking fault complexes $\left(\mathrm{Si}_{\mathrm{C}-\mathrm{SF})}{ }^{19,20}\right.$ and tiny polytype inclusions. ${ }^{21}$ These point defects in the crystal lattice of silicon carbide can be excited optically and emit single photons on demand. ${ }^{10-12,16,20,22}$ However, the greatest advantage of silicon carbide over diamond and recently emerged $2 \mathrm{D}$ materials $\mathrm{s}^{23,24}$ is its semiconductor properties. $\mathrm{SiC}$ can be efficiently doped with both donors and acceptors and demonstrate distinct electron and hole conductivity. ${ }^{1,25}$ Moreover, $\mathrm{SiC}$ devices can be fabricated in a well-developed CMOS compatible process flow. ${ }^{1}$ These advantages are very promising for the design and development of practical electrically-driven devices for quantum information and communication systems, particularly for the development of electrically pumped singlephoton sources ${ }^{26}$ which can operate at room temperature. Implementation of electrical pumping is essentially important for practical single-photon sources since optically pumped devices are much less energy efficient and are more difficult to be integrated on a chip. ${ }^{27}$ Another advantage of silicon carbide is that $\mathrm{SiC}$ is an indirect bandgap semiconductor material, which ensures a low background luminescence level due to radiative band-to-band transitions, which are unavoidable in electricallypumped devices at high injection levels. The result of the recent intense research efforts was the demonstration of single-photon emission from color centers in silicon carbide under electrical pumping at room temperature. ${ }^{19}$ The measured brightness of these emitters was higher than that of the electrically pumped color centers in diamond ${ }^{28-30}$ and zinc oxide, ${ }^{31}$ as well as quantum $\operatorname{dots}^{32}$ at room temperature, but lower than that of the optically pumped color centers. ${ }^{22,33}$ Further research aimed at developing practical single-photon sources urgently needs an understanding of the physics behind the process of photon emission from a single color center in $\mathrm{SiC}$ under electrical pumping and knowledge about how the demonstrated record emission characteristics can be further improved.

Here, we perform a rigorous theoretical and numerical study of single-photon electroluminescence of color centers in a silicon carbide diode and predict the photon emission rate and the correlation between emitted photons. Using a comprehensive computational approach, we perform 2D numerical simulations of the $\mathrm{p}^{+}-\mathrm{n}-\mathrm{n}^{+} 4 \mathrm{H}-\mathrm{SiC}$ single-photon emitting diode. We demonstrate a highly nonlinear dependence of the photon emission rate on the pump current, which can unexpectedly turn into a linear

\footnotetext{
'Laboratory of Nanooptics and Plasmonics Moscow Institute of Physics and Technology, Dolgoprudny 141700, Russian Federation

Correspondence: Dmitry Yu. Fedyanin (dmitry.fedyanin@phystech.edu)
}

Received: 12 August 2017 Revised: 5 January 2018 Accepted: 19 January 2018

Published online: 21 February 2018 

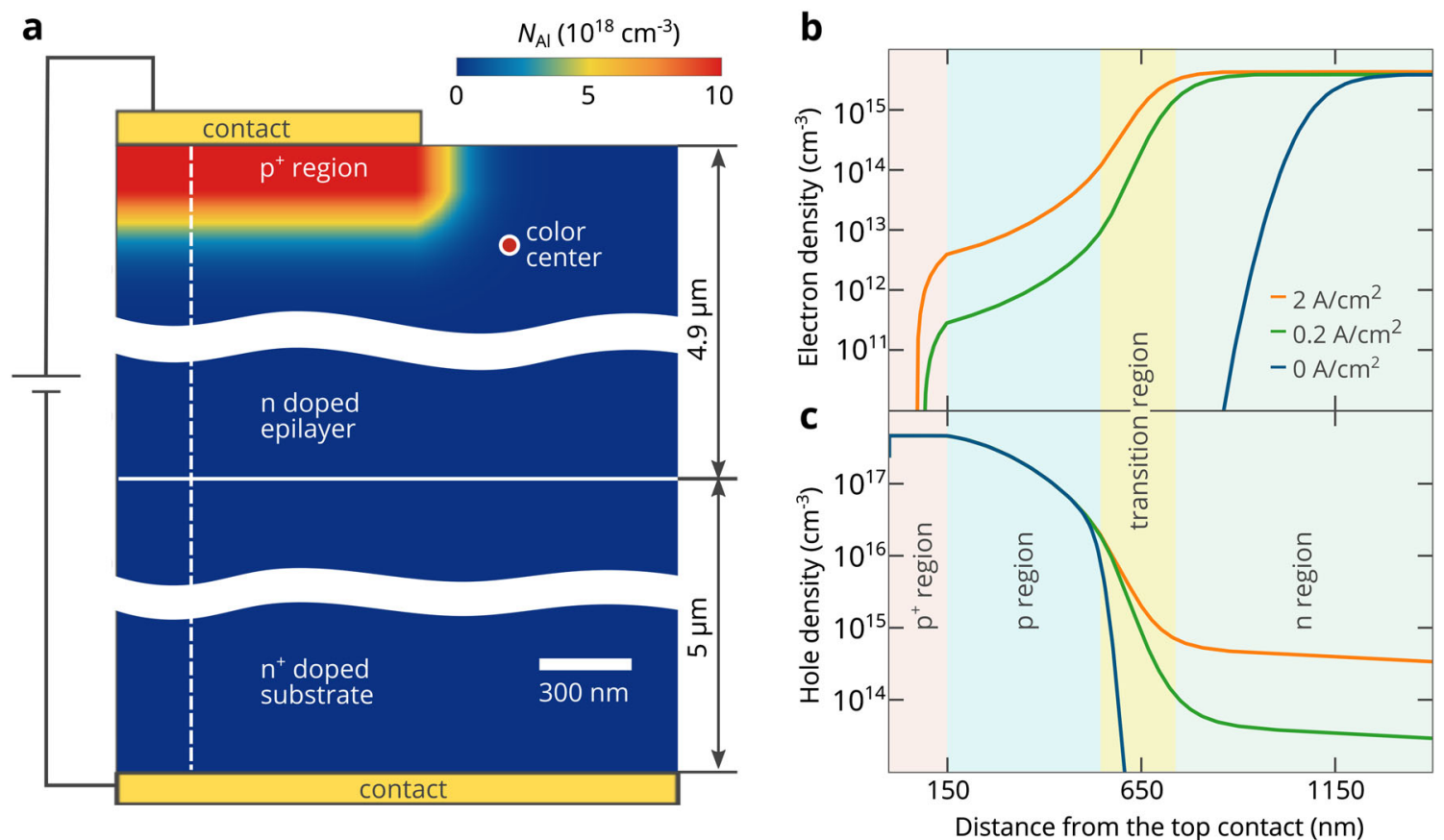

Fig. 1 a Schematic of the $\mathrm{p}^{+}-\mathrm{n}-\mathrm{n}^{+} \mathrm{SiC}$ diode with a color center in the $\mathrm{n}$-type layer. The 2D map shows the acceptor doping profile, which reproduces the experimental conditions in ref. ${ }^{19}$. b, c Simulated spatial distributions of electrons (a) and holes (b) along the dashed line shown in panel (a)

(unsaturated) dependence at high pump currents due to selfheating effects. Our numerical simulations accurately reproduce the triple exponential second-order autocorrelation functions obtained in the experiment in a broad range of injection currents. Finally, we propose and simulate an optimized $p-i-n$ singlephoton emitting diode. This diode can give the possibility to increase the photon emission rate by four orders of magnitude compared to what was observed in the experiments and design a gigahertz single-photon source which does not require cooling and operates under ambient conditions.

\section{RESULTS}

Single-photon emitting diode on silicon carbide

We focus on the silicon antisite defect $\left(\mathrm{Si}_{\mathrm{C}}\right)$ in $4 \mathrm{H}-\mathrm{SiC}^{34}$ near or inside the stacking faults (SFs) ${ }^{35}$ (hereafter, this defect is referred as the $\mathrm{Si}_{\mathrm{C}}-\mathrm{SF}$ defect). From this defect, bright single-photon emission under electrical pumping was recently experimentally observed. $^{19,20}$ The radiative transition in the neutrally charged ${ }^{36}$ $\mathrm{Si}_{\mathrm{C}}-\mathrm{SF}$ defect produces a photon polarized in the basal plane of $4 \mathrm{H}-\mathrm{SiC}_{1}^{19}$ which promotes the collection efficiency and gives the possibility to achieve a high brightness of the single-photon source. Despite that most of our calculations and simulations are performed for the $\mathrm{Si}_{C}$-SF defect, we claim that our analysis can be readily used for any electrically pumped color center in silicon carbide since the electrical properties of the system are determined by the SiC crystal rather than by the point defect in the crystal lattice.

Since the process of electroluminescence of the color center essentially involves interaction of the color center with the crystal, namely electron and hole exchange, we start with the electron and hole transport in the $\mathrm{p}^{+}-\mathrm{n}-\mathrm{n}^{+} 4 \mathrm{H}-\mathrm{SiC}$ diode, which contains a color center in the n-type region of the structure (see Fig. 1a). Such a geometry was recently used to design an electrically pumped single-photon source. ${ }^{19}$ The $\mathrm{n}^{+}$substrate is doped with nitrogen at a concentration of $7 \times 10^{19} \mathrm{~cm}^{-3}$. The concentration of donors in the n-type epilayer is much lower and is equal to $4 \times 10^{15} \mathrm{~cm}^{-3}$.
The p-type region obtained by implantation of aluminum in the epilayer features a nonuniform distribution of acceptors, which is quantitatively shown in Fig. 1a. To find densities of electrons and holes, which govern the electron and holes exchange rates between the color center and the SiC crystal, we have performed self-consistent 2D finite difference simulations of carrier transport (details are provided in the Methods section and in Supplementary Methods).

Figure $1 \mathrm{~b}, \mathrm{c}$ shows the distribution of electrons and holes in equilibrium and at high injection levels. Whereas in equilibrium there are no holes in the n-type region, the density of holes reaches $10^{14}-10^{15} \mathrm{~cm}^{-3}$ at high injection current densities at the top contact (hereafter, referred simply as the injection current density). At the same time, electrons start to penetrate deep into the p-type region, as the bias voltage increases (see Fig. 1b). In Fig. $1 \mathrm{~b}, \mathrm{c}$, we can introduce an additional region. In this transition region, there are no electrons and holes in equilibrium. At the same time, at high injection levels, one can simultaneously create high densities of both electrons and holes in this region. Such a region should always exist in $\mathrm{p}-\mathrm{n}$ junctions, and its position and width depend on the doping profile. As shown below, the transition region is crucially important for achieving a high rate of single-photon emission. The electron and hole exchange rates between the color center and the semiconductor crystal are determined by the electron and hole capture processes and are therefore governed by the densities of the corresponding charge carriers.

Physics of the electroluminescence process

Similar to the NV center in diamond, ${ }^{37}$ the $\mathrm{Si}_{\mathrm{C}}-\mathrm{SF}$ defect in $4 \mathrm{H}-\mathrm{SiC}$ has at least two charge states. ${ }^{19,36}$ This requirement is essential for electroluminescence since the process of electron and hole exchange is accompanied by the change in the charge of the color center. One of these states is positively charged, and one is neutral (see Fig. 2a). In principle, a negatively charged state can also exist (Fig. 2a), making the process of single-photon electroluminescence more complicated (for details, see 
a positively charged

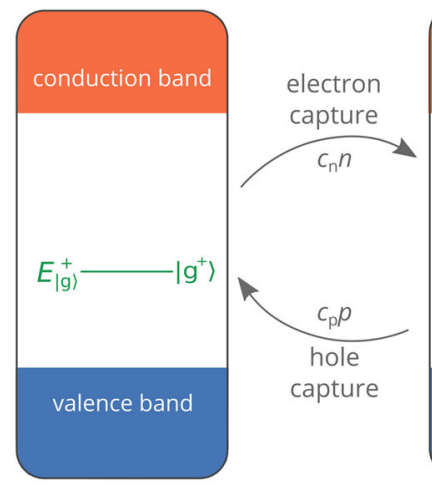

neutral

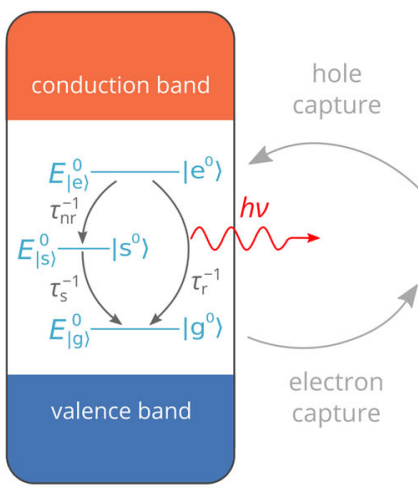

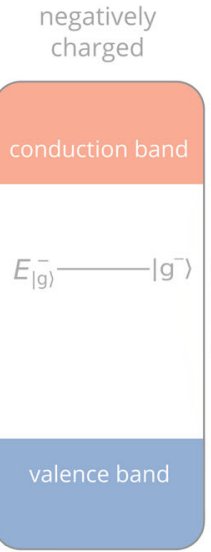

b

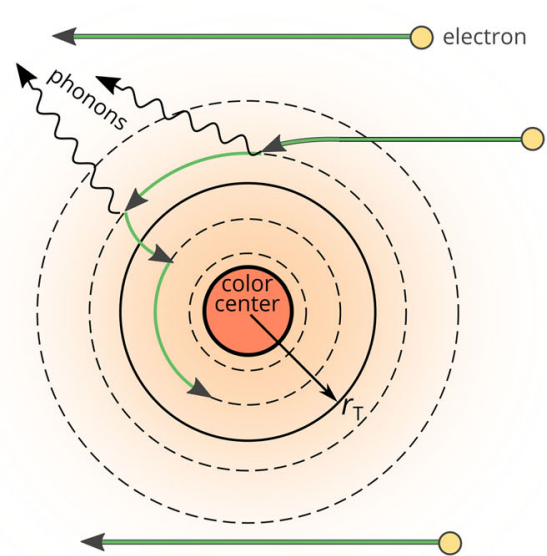

Fig. 2 a Schematic diagram of the single-photon electroluminescence process for the $\mathrm{Si}_{C}-\mathrm{SF}$ defect in $4 \mathrm{H}-\mathrm{SiC}$. $\left|\mathrm{e}^{0}>,\right| \mathrm{g}^{0}>, \mid s^{0}>$ denote the excited, ground, and shelving states of the neutral $\mathrm{Si}_{C}-\mathrm{SF}$ center, respectively. $\lg ^{+}>$is the only (ground) state of the positively charged $\mathrm{Si}_{C}-\mathrm{SF}$ center. Arrows indicate transitions among different states of the color center. Transition rates are shown next to each arrow. $\mathbf{b}$ Illustration of the electron capture process by the positively charged color center. $r_{\mathrm{T}}$ is the Thomson radius

Supplementary Methods). However, theoretical studies of charge states of the $\mathrm{Si}_{\mathrm{C}}$ defect $\left(\mathrm{D}_{1}\right.$ center) in $4 \mathrm{H}-\mathrm{SiC}_{1}^{34}$ which has a similar structure, ${ }^{36}$ do not reveal the presence of such a state. ${ }^{38}$ Since the photoluminescence measurements do not show a noticeable difference between defects in the n-type and p-type silicon carbide, ${ }^{19}$ we can conclude that the defect has a deep-donor-like nature and, consequently, it is not ionized in either n-type or ptype silicon carbide unless the dopant concentration reaches $\sim 10^{20} \mathrm{~cm}^{-3}$. Therefore, the observed single-photon photoluminescence spectrum can be attributed only to the transitions between the ground and excited states of the neutrally charged $\mathrm{Si}_{C}-\mathrm{SF}$ center (see Fig. 2a).

Figure $2 \mathrm{a}$ shows the schematic diagram of the single-photon electroluminescence for the $\mathrm{Si}_{\mathrm{C}}-\mathrm{SF}$ center. In the steady state, the process is cyclic. Therefore, let us consider the $\mathrm{Si}_{\mathrm{C}}$-SF center in the neutral ground state $\mid \mathrm{g}^{0}>$ right after photon emission. The color center in the neutral ground state can either capture a hole from the valence band and become positively charged or capture an electron from the conduction band and become negatively charged. Since according to the DFT studies, ${ }^{38}$ the negatively charged state does not exist, the $\mathrm{Si}_{C}$-SF center can capture only a hole. Here, we assume that there is only a one energy state $\left|\mathrm{g}^{+}\right\rangle$in the positively charged $\mathrm{Si}_{C}-\mathrm{SF}$ center. The hole capture rate can be found as $c_{p} p$, where $p$ is the density of holes in the vicinity of the color center. The hole capture rate constant $c_{p}$ is given by $c_{p}=$ $\sigma_{\mathrm{p}} u_{\mathrm{p}}$, where $u_{\mathrm{p}}$ is the average thermal velocity of holes in silicon carbide, and $\sigma_{\mathrm{p}}$ is the capture cross-section by the neutral $\mathrm{Si}_{\mathrm{C}}-\mathrm{SF}$ center. $\sigma_{\mathrm{p}}$ is estimated to be about $5 \times 10^{-15} \mathrm{~cm}^{2} .39,40$ We should note that in principle, a hole can be captured into some excited state and then experience relaxation to the ground state $\left|g^{+}\right\rangle$. However, such nonradiative relaxations are typically orders of magnitude faster than the hole capture rate ${ }^{41}$ and can therefore hardly be noticed in the electroluminescence measurements. The electroluminescence studies do not indicate the existence of radiative transitions among excited and ground levels of the positively charged $\mathrm{Si}_{\mathrm{C}}-\mathrm{SF}$ center. ${ }^{19}$ Hence, only one energy state $\mathrm{g}^{+}>$in the positively charged $\mathrm{Si}_{\mathrm{C}}-\mathrm{SF}$ center can be considered.

The positively charged center attracts negatively charged electrons and can capture one of them from the conduction band. This process changes the charge of the $\mathrm{Si}_{\mathrm{C}}-\mathrm{SF}$ center from positive to neutral $\left(\left|g^{+}>\rightarrow\right| e^{0}>\right.$ transition in Fig. 2a). The electron capture rate is equal to the product of the electron capture constant $c_{n}$ and the density $n$ of free electrons in the vicinity of the color center. $c_{\mathrm{n}}=\sigma_{\mathrm{n}} U_{\mathrm{n}}$, where $\sigma_{\mathrm{n}}$ is the electron capture crosssection, and $v_{n}$ is the average thermal velocity of free electrons. The electron capture cross-section can be found as follows. The attractive potential of the color center forms a set of discrete bound levels, which can be populated by electrons from the conducting band. To be captured, an electron should lose an energy larger than $k_{\mathrm{B}} T$, otherwise, it will be released back to the conduction band by thermal excitation. According to the cascade capture model, ${ }^{42,43}$ the energy is lost due to interaction with acoustic phonons. In each act of the electron-photon interaction, the electron loses only a small portion of its kinetic energy, which defines the cascade nature of the process (see Fig. 2b). The electron capture cross-section $\sigma_{\mathrm{n}}$ can be found as a ratio of the volume of the Thomson sphere with radius $r_{\mathrm{T}}$ to the energy relaxation length due to electron-phonon interaction: ${ }^{42} \sigma_{\mathrm{n}}=$ $4 \pi r_{\mathrm{T}}^{3} /\left(3 l_{0}\right)$. The energy relaxation length $I_{0}=l k_{\mathrm{B}} T /\left(2 m_{\mathrm{eff}} s^{2}\right)$ is significantly larger than the electron mean free path / since only a small fraction of the electron kinetic energy is dissipated in each act of phonon emission. Here, $s$ is the speed of sound in $4 \mathrm{H}-\mathrm{SiC}$ and $m_{\text {eff }}$ is the effective electron mass. For the transition region in Fig. $1 b, c$, where we expect the highest photon emission rate from the color center, $\sigma_{\mathrm{n}}=6.1 \times 10^{-15} \mathrm{~cm}^{2}$ at room temperature.

In the next stage, the excited state of the neutral center relaxes to the ground state via radiative $\left(\left|e^{0}>\rightarrow\right| g^{0}>\right)$ and nonradiative transitions $\left(\left|e^{0}>\rightarrow\right| s^{0}>\rightarrow \mid g^{0}>\right.$ ) (see Fig. 2a for details). After that, the $\mathrm{Si}_{C}-\mathrm{SF}$ center appears in the neutral ground state $\mid \mathrm{g}^{0}>$ and can repeat the cycle of the electroluminescence process.

Following Fedyanin and Agio $^{37}$ and neglecting thermal excitations, we can quantitatively describe the populations of the ground and excited states of the neutral and positively charged $\mathrm{Si}_{\mathrm{C}}-\mathrm{SF}$ centers:

$\left\{\begin{array}{l}\frac{d n_{\mathrm{e}}}{d \tau}=n_{+} c_{\mathrm{n}} n-\frac{n_{\mathrm{e}}}{\tau_{\mathrm{r}}}-\frac{n_{\mathrm{e}}}{\tau_{\mathrm{nr}}} \\ \frac{d n_{\mathrm{s}}}{d \tau}=\frac{n_{\mathrm{e}}}{\tau_{\mathrm{nr}}}-\frac{n_{\mathrm{s}}}{\tau_{\mathrm{s}}} \\ \frac{d n_{\mathrm{g}}}{d \tau}=\frac{n_{\mathrm{e}}}{\tau_{\mathrm{r}}}+\frac{n_{\mathrm{s}}}{\tau_{\mathrm{s}}}-n_{\mathrm{g}} c_{\mathrm{p}} p \\ \frac{d n_{+}}{d \tau}=n_{\mathrm{g}} c_{\mathrm{p}} p-n_{+} c_{\mathrm{n}} n\end{array}\right.$

In this system of equations, $n$ and $p$ are the electron and hole densities in the vicinity of the color center, respectively, $n_{\mathrm{e}}, n_{\mathrm{s}}$ and $n_{\mathrm{g}}$ are the populations of the excited, shelving and ground states of the neutral defect, and $n^{+}$is the population of the positively charged state. $1 / \tau_{r}, 1 / \tau_{n r}, 1 / \tau_{s}$ are the transition rates between the excited, shelving, and ground states of the neutral center. From the photoluminescence measurements, ${ }^{19} \tau_{\mathrm{r}}$ and $\tau_{\mathrm{nr}}$ are estimated to be $3.6 \mathrm{~ns}$ and $36 \mathrm{~ns}$, respectively. The lifetime of the excited state equals $\tau_{\mathrm{e}}=1 /\left(1 / \tau_{\mathrm{r}}+1 / \tau_{\mathrm{nr}}\right)=3.3 \mathrm{~ns}$. The lifetime of the shelving state estimated from the experimentally measured $g^{(2)}$ functions ${ }^{19}$ is found to be about 35 ns. For the complete list of 
a

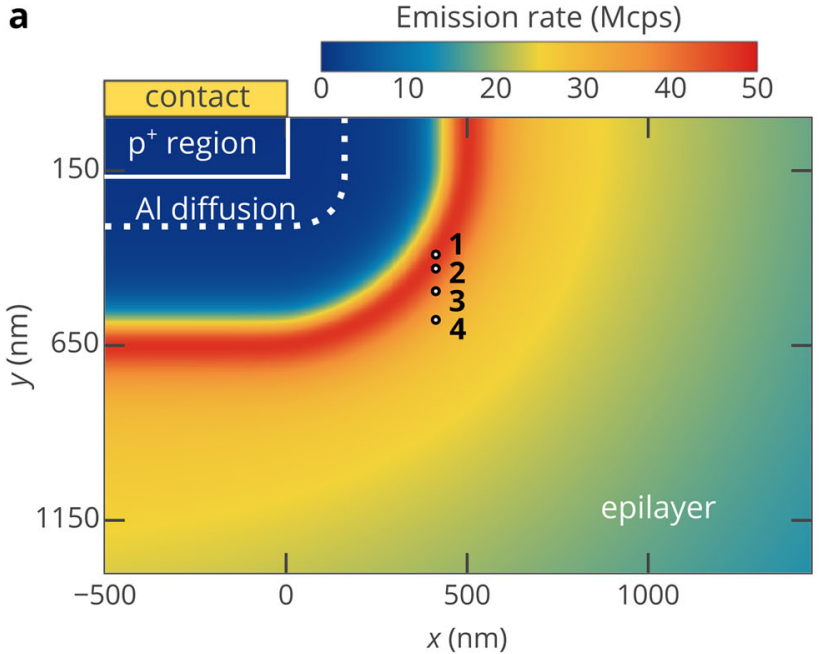

C

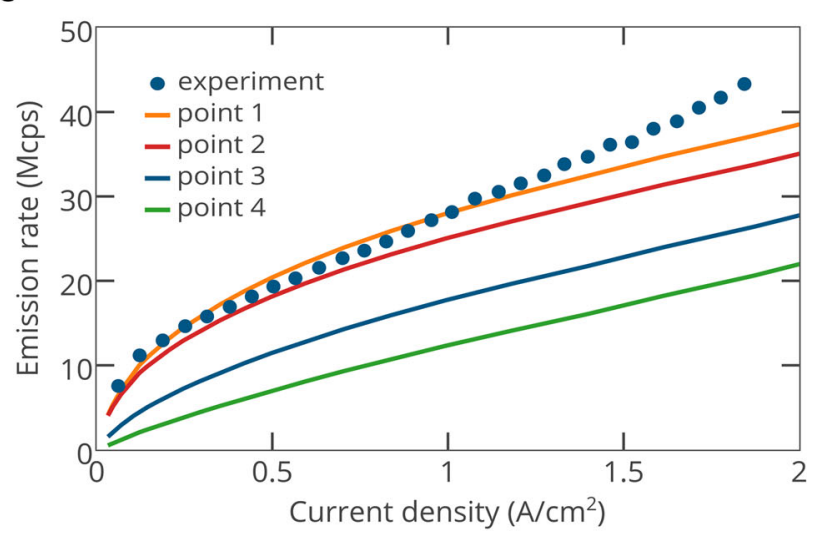

b

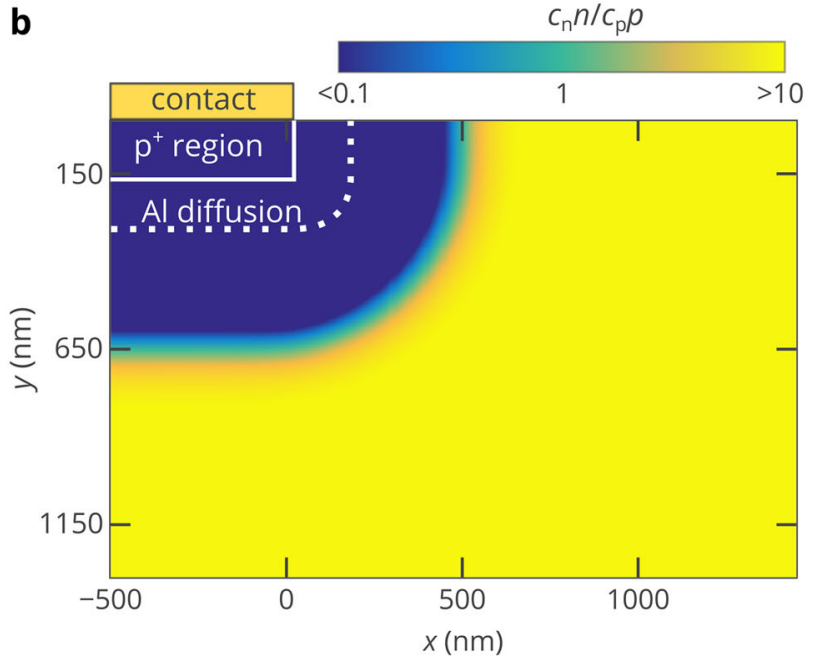

d

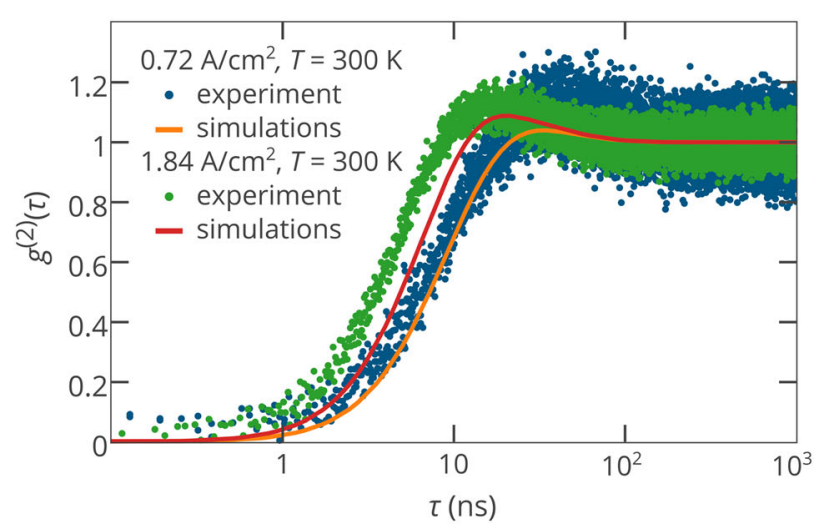

Fig. 3 a Dependence of the single-photon emission rate from the $\mathrm{Si}_{\mathrm{C}}-\mathrm{SF}$ center on its position within the $\mathrm{SiC}$ diode at a pump current density of $1.84 \mathrm{~A} / \mathrm{cm}^{2}$, which corresponds to the maximum current of $14 \mathrm{~mA}$ in the experiment. ${ }^{19} \mathbf{b}$ The ratio of the electron capture rate to the hole capture rate at a pump current density of $1.84 \mathrm{~A} / \mathrm{cm}^{2}$. c Photon emission rate as a function of the current density at points marked in panel (a). The experimental curve is retrieved from ref. ${ }^{19}$ and corrected for the detection efficiency (53\%) and collection efficiency (estimated to be $1.5 \%$, which is slightly lower than the value reported in ref. $\left.{ }^{19}\right)$. (d) $g^{(2)}$ curves retrieved from ref. ${ }^{19}$ and numerically simulated for the color center at point 2 in panel a

parameters used in the calculations, see Supplementary Methods. Solving Eq. (1) in the steady state, we can find the single-photon emission rate:

$R=\frac{n_{\mathrm{e}}^{\mathrm{ss}}}{\tau_{\mathrm{r}}}=\left[\left(\frac{1}{c_{\mathrm{n}} n}+\frac{1}{c_{\mathrm{p}} p}\right)\left(1+\frac{\tau_{\mathrm{r}}}{\tau_{\mathrm{nr}}}\right)+\tau_{\mathrm{r}}\left(1+\frac{\tau_{\mathrm{s}}}{\tau_{\mathrm{nr}}}\right)\right]^{-1}$

where $n_{\mathrm{e}}^{\text {ss }}$ denotes the steady-state population of the excited state of the neutral defect. Expression in the square brackets contains two terms. The first term represents electrical pumping, while the second is responsible for the transitions from the excited to the ground state. It can be clearly seen that the long-lived shelving state can substantially affect the photon emission rate, but only at very high injection levels.

\section{Single-photon emission upon electrical injection}

Using the results of the electron and hole transport simulations, we can quantitatively evaluate the photon emission rate from the $\mathrm{Si}_{\mathrm{C}}-\mathrm{SF}$ center in the silicon carbide diode depicted in Fig. 1a. Figure $3 a$ shows the dependence of the photon emission rate on the position of the $\mathrm{Si}_{C}-\mathrm{SF}$ defect in the $\mathrm{p}^{+}-\mathrm{n}-\mathrm{n}^{+} \mathrm{SiC}$ diode at a high injection level. The emission rate depends strongly on the distance from the edge of the $\mathrm{p}^{+}$region. The simulations demonstrate that only defects in a narrow region can give bright single-photon emission, which was observed in the experiment. Remarkable is that the width of this region is about $150 \mathrm{~nm}$ and it coincides with the transition region shown in Fig. 1b, c. As follows from Eq. (2), bright photon emission requires the density of both electrons and holes to be significantly high. This requirement can be satisfied only in the transition region, which is clearly seen in Fig. 3b, which shows the ratio between the electron and hole capture rate. In this figure, one can identify two areas, where either the electron capture (blue region) or hole capture (yellow region) process is slow. In between these areas, the electron and hole capture rates are nearly equal to each other.

Figure $3 c$ shows the numerically simulated and experimentally measured dependences of the single-photon emission rate on the pump current. One can see that the plotted curves are nonlinear, and the emission rate is not directly proportional to the pump current. We should emphasize that this nonlinearity is not due to saturation. The photon emission rate is indirectly related to the pump current via the carrier densities. In turn, the carrier densities are not directly proportional to the injection current, as in most semiconductor devices. Since the position of the bright color center in the experiment is not known exactly, ${ }^{19}$ we can allow a slight variation to fit the experimental data better (Fig. 3c). At low pump currents, one can see good quantitative agreement between the theoretical (red curve) and experimental (dotted 
curve) results. However, at high pump currents, the discrepancy cannot be ignored. The theory holds that the emission rate starts to "saturate" at high currents, while in the experiment the emission rate is linearly proportional to the pump current.

To understand the nature of the difference between theory and experiment, we use additional information provided by the measurements of the correlation between photons. ${ }^{44}$ Figure $3 \mathrm{~d}$ shows the simulated $g^{(2)}$ curves (see Methods section) at moderate and high pump currents, which are plotted next to the experimental data points. First, we note that the theoretical curves ideally reproduce the triple exponential dependences, which were observed in the single-photon electroluminescence measurements. ${ }^{19}$ Lohrmann et al. ${ }^{19}$ demonstrated that the presence of the fourth high-energy level can explain such a triple exponential dependence. Here, we show that this fourth state, in fact, corresponds to the ground state of the positively charged $\mathrm{Si}_{\mathrm{C}^{-}}$ SF center rather than to any excited state of the neutral color center. At the same time, we have the same number of transitions among different states of the color center in the proposed electroluminescence model as in the conventional four-level model. The pair of the electron and hole capture processes can be considered as a two-step process that brings the color center from the neutrally charged ground state to the neutrally charged excited state (see Fig. 2a). In contrast, the one-step excitation process in the case of optical pumping stipulates the double exponential dependence in the photoluminescence measurements of $\mathrm{Si}_{\mathrm{C}}$-SF centers. ${ }^{19}$ Second, at a pump current density of $0.7 \mathrm{~A} / \mathrm{cm}^{2}$ (as well as at lower currents), the agreement between the theoretical and experimental $g^{(2)}$ curves is as good as between the corresponding emission rates. However, at a current of $1.8 \mathrm{~A} /$ $\mathrm{cm}^{2}$, one can observe a significant difference in the $g^{(2)}$ traces, which is even higher than the difference in the emission rates (see Fig. 3c).

Self-heating of the single-photon emitting diode

We attribute the observed discrepancy in the photon emission rates and $g^{(2)}$ curves between theory and experiment to the change in the properties of the single-photon emitting diode at high currents since our simulations accurately reproduce the experimentally measured data at low injection levels. As the pump current increases, the power dissipated by the diode increases, which gives rise to the device temperature. This was indirectly confirmed in the experiment by the observed displacement (probably caused by thermal expansion) of the color center at high pump currents. ${ }^{38}$ Therefore, we proceed with the evaluation of the impact of self-heating on the photon emission rate and the dynamics of single-photon emission. Heat is generated due to the nonradiative recombination in the bulk of the diode and at the top and bottom contacts. ${ }^{45}$ Due to the high thermal conductivity of $\mathrm{SiC}_{1}^{39}$ the temperature distribution across the sample should be homogeneous. The net heating power is approximately equal to the power $J V$ supplied to the diode. In turn, the temperature rise is proportional to the heating power. At high injection levels, the diode operates well above the turn-on voltage ${ }^{19}$ and the heating power increases proportionally to the current squared. The temperature rise significantly increases the electron and hole densities due to the relatively high activation energies of donors (nitrogen) and acceptors (aluminum) in SiC. The hole capture rate constant $c_{\mathrm{p}}$ also increases due to the increase in the hole thermal velocity. At the same time, the temperature dependence of the electron capture rate constant $c_{n}$ is more complicated. Whereas the electron thermal velocity increases as $T^{1 / 2}$, the electron capture cross-section is inversely proportional to $\sim T^{2}$. Thus, $c_{n}$ decreases with temperature increase. Nevertheless, our numerical simulations in a wide temperature range show that the temperature rise increases the brightness of the single-photon emitting diode (Fig. 4a), which is also confirmed by the reduction
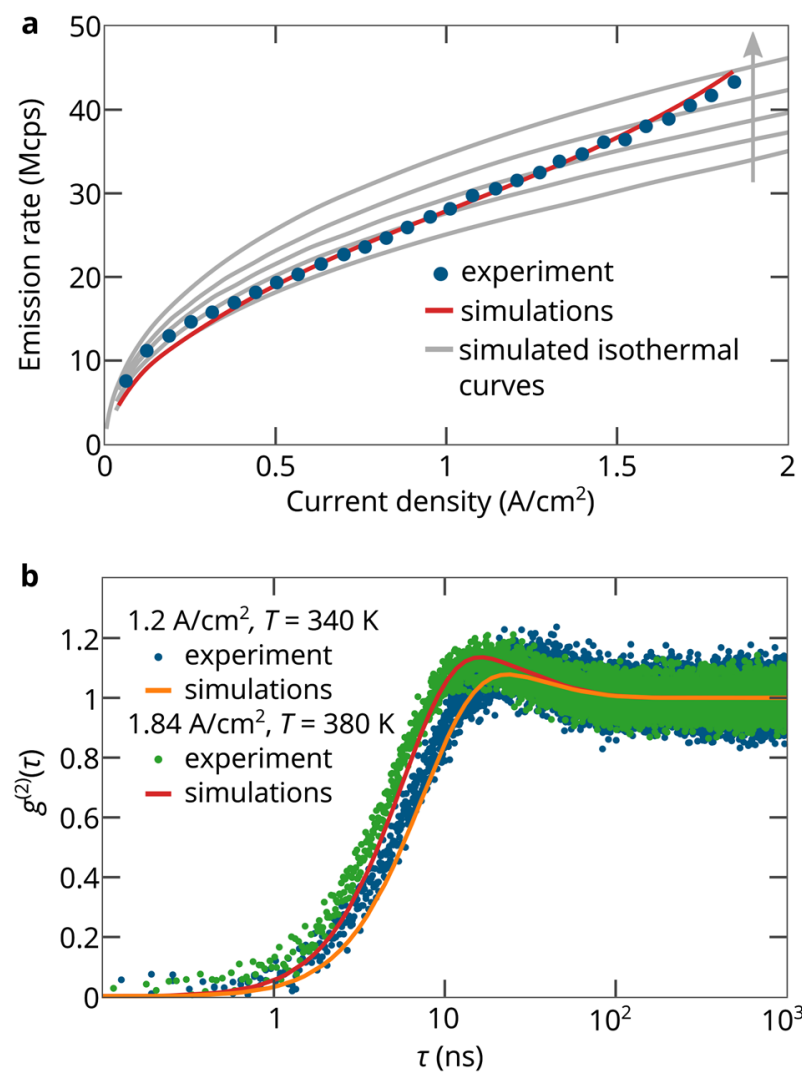

Fig. 4 a Photon emission rate versus pump current for the color center at point 2 in Fig. 3a. The red curve is simulated taking into account the self-heating effect, while the grey curves are isothermal curves at $300,320,340,360$, and $380 \mathrm{~K}$ (the grey arrow shows the direction of temperature increase). The experimental data are the same as in Fig. 3c. b $g^{(2)}$ curves retrieved from ref. ${ }^{19}$ and numerically simulated for the color center at point 2 in Fig. 3a

of the characteristic time of the $g^{(2)}$ function (Fig. 4b). The results are consistent with our assumption that the temperature rise is proportional to the pump current squared: at a current density of $1.2 \mathrm{~A} / \mathrm{cm}^{2}$ the heating power is twice lower than at $1.84 \mathrm{~A} / \mathrm{cm}^{2}$, so is the temperature rise with respect to the ambient temperature. The numerically simulated curves (Fig. 4) nearly ideally reproduce the experimentally measured data demonstrating that the developed theoretical model accurately describes the photophysics of electrically pumped $\mathrm{Si}_{C}-\mathrm{SF}$ centers.

Towards an electrically driven gigahertz single-photon source The experimentally demonstrated characteristics of the SiC singlephoton emitting diode are, to the best of our knowledge, superior to any electrically driven system at room temperature. However, they still should be improved to meet the requirements of modern and future quantum technology applications. Particularly, for practical secure quantum communications, the photon emission rate should be in the gigahertz range. Can this be achieved with electrical control?

The photon emission rate is limited by three factors: (1) the lifetime of the excited state, (2) the quantum efficiency and the lifetime of the shelving state, and (3) the electron and hole capture rates. The radiative lifetime of the excited state of the neutrally charged $\mathrm{Si}_{C}-\mathrm{SF}$ center, the lifetime of the shelving state and quantum efficiency virtually limit the maximum emission rate to $0.1 \mathrm{Gcounts} / \mathrm{s}$ as follows from Eq. (2). However, the radiative lifetime $\tau_{r}$ can be significantly reduced using an optical 
nanoantenna, ${ }^{46-50}$ which increases the rate of radiative transitions due to the Purcell effect. In addition, the nanoantenna approach can give the possibility to improve the collection efficiency by more than an order of magnitude. ${ }^{16}$ The Purcell factor of about 50 can be relatively easily achieved in most nanoantenna geometries, which reduces the radiative lifetime of the excited state to $70 \mathrm{ps}$. Therefore, we only need to increase the carrier capture rates to $(1-10) \times 10^{9} \mathrm{~s}^{-1}$, which, however, cannot be done straightforwardly.

Silicon carbide is a wide-bandgap semiconductor, and consequently, the activation energies of donor $(\sim 0.05 \mathrm{eV}$ for nitrogen on hexagonal sites and $\sim 0.10 \mathrm{eV}$ on cubic sites $)^{51}$ and acceptors $(\sim 0.19 \mathrm{eV} \text { for aluminum })^{52}$ are much higher than in silicon or gallium arsenide. For this reason, the densities of electrons and holes are about 30 times lower than the concentration of the doping impurities. Moreover, in the active region of $p-n$ and $p-i-n$ diodes, where it is possible to create a high density of both electrons and holes, the densities of carriers are 100-10,000 times lower than in the $\mathrm{p}$ - and $\mathrm{n}$-type regions. The higher the doping level, the higher the difference. Our simulations show that in the studied geometry (see Fig. 1a), the photon emission rate is limited to about 70 Mcounts $/ \mathrm{s}$ at current densities below $5 \mathrm{~A} / \mathrm{cm}^{2}$ at room temperature.

To significantly increase the photon emission rate, we need to change the design of the single-photon emitting diode. First, the doping level should be increased. In this case, the density of free carriers in the $p$ - and n-type regions increase not only due to the increased dopant concentrations but also due to the reduction in the activation energies of dopants. ${ }^{52,53}$ Second, the size of the lightly doped region should be decreased, since the density of holes rapidly decreases with the distance from the p-type region due to recombination in the active region and the potential barrier at the $\mathrm{p}-\mathrm{i}-\mathrm{n}$ junction. Similarly, the density of electrons decreases with the distance from the n-type region. Therefore, the $p-n$ structure is preferred over the $p-i-n$ structure. However, in this case, bright photon emission can be obtained only from a color center placed in a very narrow region at the $p-n$ junction. To avoid this limitation, we propose a $\mathrm{p}-\mathrm{i}-\mathrm{n}$ diode with a graded doping profile in the narrow i-region, which can be achieved by both CVD growth and ion implantation (Fig. 5a).

Figure $5 b$ shows the dependence of the maximum photon emission from the $\mathrm{Si}_{C}$-SF center in the proposed diode configuration. One can see that at a current density of $5 \mathrm{~A} / \mathrm{cm}^{2}$, the carrier capture processes limit the photon emission rate to about 13 Gcount/s, which is above the limit imposed by the radiative lifetime $\tau_{\mathrm{r}}$ of the excited state in a bulk material. However, using the Purcell effect in optical nanoantennas, $\tau_{r}$ can be reduced by a factor of ${ }^{46-49,54} 50-200$ and a photon emission rate of about 5-10 Gcounts/s is practically achievable. Here, we should emphasize that the operating temperature is approximately equal to $300 \mathrm{~K}$, since the heating power cannot noticeably increase the device temperature if the size of the contact is less than $20 \mu \mathrm{m} .{ }^{45}$ Finally, we note that our theoretical studies demonstrate that the photon emission rate might be further increased to above $15 \mathrm{Gcount} / \mathrm{s}$ using a properly designed donor doping profile. However, in this case, one needs novel nanoantennas with record characteristics, which can reduce the radiative lifetime of the excited state of the $\mathrm{Si}_{C}-\mathrm{SF}$ center by a factor of 1000 .

\section{DISCUSSION}

In this work, we have presented a theoretical description of emission properties of electrically-pumped $\mathrm{Si}_{\mathrm{C}}-\mathrm{SF}$ color centers in silicon carbide. The combined study of transitions between states of the $\mathrm{Si}_{C}$-SF center and the electron and hole transport in silicon carbide have revealed the photophysics of the color center electroluminescence. It has been shown that the photon emission process is mostly determined by the electron and hole capture
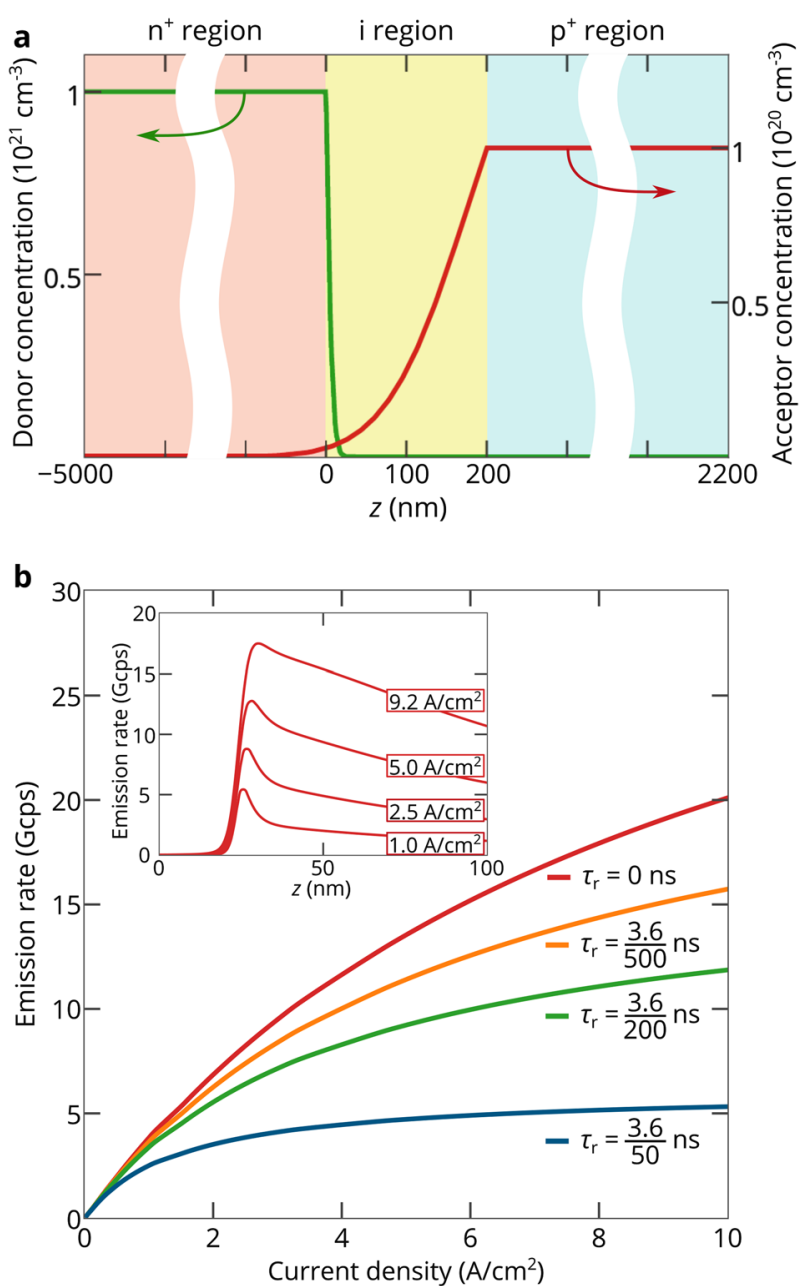

Fig. 5 a Schematic illustration of the proposed $p-i-n$ diode with a graded doping profile in the i-region and the distributions of donors and acceptors. $\mathbf{b}$ Maximum rate of photons emitted by the $\mathrm{Si}_{C}-\mathrm{SF}$ center versus pump current at room temperature for the diode shown in panel (a). Simulations are performed for different values of the radiative lifetime of the excited state of the $\mathrm{Si}_{\mathrm{C}}-\mathrm{SF}$ center which can be significantly decreased using the Purcell effect in nanoantennas. The red curve corresponds to the maximum possible photon emission rate limited by the electron and hole capture processes. Inset: Maximum possible photon emission rate as a function of the position of the color center in the $\mathrm{p}-\mathrm{i}-\mathrm{n}$ diode for different pump currents

processes. Therefore the obtained results can be applied with no change to any color center in $4 \mathrm{H}-\mathrm{SiC}$ which has neutral and positively charged states, since the electron capture rate by the positively charged defect is determined by the properties of the semiconductor material (sound velocity, electron mobility, and density of free electrons) rather than by the internal structure of the defect. At the same time, the hole capture rate by the neutral defect depends on the defect structure. Nevertheless, for most defects, the capture cross-section is roughly equal to the lattice constant of silicon carbide. ${ }^{39,40}$ We also extended our model to color centers with three charge states (negative, neutral, and positive), see Supplementary Methods for details. With minimal changes, this model can be applied to most color center in silicon carbide, such as the $C_{S_{i}} V_{C}$ center. Here we should emphasize that multiple charge states, such as $(+2),(+3)$ or $(-2)$ states, cannot be efficiently excited electrically. The problem is that the singlycharged defect is an attractive center for one type of carriers and 
is a repulsive center for the other type of carriers. For example, the positively charged defect attracts electrons and repels holes. The capture cross-section for electrons is in the range from $10^{-15}$ to $10^{-13} \mathrm{~cm}^{2}$, while the capture cross-section for holes is roughly 4-5 orders of magnitude lower. Hence, the photon emission rate from a multiple charge state is expected to be much lower than from a single-charged/neutral state. Nevertheless, silicon carbide can be easily doped with both donors and acceptors, and high densities $\left(>10^{18} \mathrm{~cm}^{-3}\right)$ of free electron and holes can be created, ${ }^{52,53}$ which provides favorable conditions for electrical pumping of even multiple charge states. This property gives silicon carbide a distinct advantage over diamond (a well-recognized host material for single color centers). Since it is not possible to create a high density of both electrons and holes in diamond under ambient conditions, ${ }^{37,55}$ color centers in diamond cannot be as bright as in silicon carbide.

Our numerical simulations based on the developed theoretical model accurately reproduce the experimental data and explain the recently observed features of silicon carbide single-photon emitting diodes, which include the distinct triple exponential behavior of the second-order autocorrelation function and the linear (unsaturated) dependence of the emission rate on the pump current at high injection levels. We found that the latter is caused by diode self-heating, which cannot be ignored at high pump currents. Similar to diamond, ${ }^{37}$ due to the high activation energy of acceptors in silicon carbide, the temperature rise increases the density of free carriers and consequently the photon emission rate. However, the effect is not that strong as in diamond and heating cannot be used to increase the brightness by orders of magnitude. At the same time, self-heating can ensure a linear (unsaturated) growth of the emission rate in a broad range of pump currents despite that the dependence of the carrier densities, which govern photon emission, on the pump current is highly nonlinear. Excellent quantitative agreement between theoretical and experimental curves demonstrates that the developed theoretical approach can be efficiently used in the development of novel single-photon sources. Our theory predicts that as soon as the lifetime of the excited state is decreased below a few tens of picoseconds (which can be achieved with an optical cavity or a nanoantenna), the photon emission rate is governed only by the electron and hole capture processes by the color center. We demonstrate that by using a properly designed $p-i-n$ diode structure and optical cavities, the brightness can be increased by more than four orders of magnitude compared to what was observed in the experiments. Our theoretical findings show that the photon emission rate can be as high as 10 Gcounts/ $s$ at room temperature, which makes silicon carbide single-photon emitting diodes highly advantageous over other single-photon devices for diverse practical quantum optics applications, such as unconditionally secure data communication based on quantum cryptography.

\section{METHODS}

Simulation of the single-photon emitting diode

The $2 \mathrm{D}$ numerical simulations of the silicon carbide diode were performed using the self-consistent steady-state model, which comprises Poisson's equation for the electric field and carrier densities, semiconductor driftdiffusion equations for electrons, and holes and carrier continuity equations. These differential equations supplemented with the appropriate boundary conditions at the top and bottom ohmic contacts were solved using the nextnano++ software (for details, see Supplementary Methods). The single-photon emission rate and the second-order autocorrelation function were calculated using the obtained carrier density distributions and the developed theoretical framework.
Evaluation of the $g^{(2)}$ function of the electrically pumped color center

The second-order autocorrelation function $g^{(2)}(\tau)$ of the $\mathrm{Si}_{\mathrm{C}}-\mathrm{SF}$ center in $4 \mathrm{H}-$ $\mathrm{SiC}$ was calculated by solving Eq. (1) with the initial conditions $n_{\mathrm{g}}=1, n_{\mathrm{e}}=$ $n_{\mathrm{s}}=n_{+}=0 .{ }^{44}$ The obtained temporal dynamics of the $\mathrm{Si}_{-}-\mathrm{SF}$ center is related to the $g^{(2)}$ function as $g^{(2)}(\tau)=n_{\mathrm{e}}(\tau) / n_{\mathrm{e}}^{\text {ss }}$, where $n_{\mathrm{e}}^{\text {ss }}$ is the steadystate population of the excited state of the neutrally charged center.

Code availability

The nextnano++ software used in the simulations is available at http:// www.nextnano.de/customer/download.php.

\section{Data availability}

All relevant data are available in Supplementary Information and from the authors upon request.

\section{ACKNOWLEDGEMENTS}

We greatly acknowledge Dr. B. C. Johnson for providing the raw experimental data. The work is supported by the Russian Science Foundation (17-79-20421).

\section{AUTHOR CONTRIBUTIONS}

I.A.K. performed the numerical simulations of the single-photon emitting diode. A.A. V. and I.A.K. carried out the calculations. D.Y.F. conceived the idea and developed the theoretical framework. All authors discussed the results and contributed to manuscript preparation.

\section{ADDITIONAL INFORMATION}

Supplementary information accompanies the paper on the npj Quantum Information website (https://doi.org/10.1038/s41534-018-0066-2).

Competing interests: The authors declare no competing interests.

Publisher's note: Springer Nature remains neutral with regard to jurisdictional claims in published maps and institutional affiliations.

\section{REFERENCES}

1. Zhe, C. F Silicon Carbide: Materials, Processing \& Devices. (CRC Press: New York, 2003).

2. Round, H. J. A note on carborundum. Electr. World 49, 309 (1907).

3. Losev, O. V. Luminous carborundum [silicon carbide] detector and detection with crystals. Telegr. i Telef. Bez. Provodov 44, 485-494 (1927).

4. Zheludev, N. The life and times of the LED - a 100-year history. Nat. Photonics 1, 189-192 (2007).

5. Aharonovich, I. et al. Diamond-based single-photon emitters. Rep. Prog. Phys. 74, 076501 (2011).

6. Pezzagna, S., Rogalla, D., Wildanger, D., Meijer, J. \& Zaitsev, A. Creation and nature of optical centres in diamond for single-photon emission-overview and critical remarks. New J. Phys. 13, 035024 (2011).

7. Aharonovich, I. \& Neu, E. Diamond nanophotonics. Adv. Opt. Mater. 2, 911-928 (2014).

8. Childress, L., Walsworth, R. \& Lukin, M. Atom-like crystal defects: from quantum computers to biological sensors. Phys. Today 67, 38-43 (2014).

9. Lounis, B. \& Orrit, M. Single-photon sources. Rep. Prog. Phys. 68, 1129-1179 (2005).

10. Castelletto, S. et al. A silicon carbide room-temperature single-photon source. Nat. Mater. 13, 151-156 (2014).

11. Castelletto, S. et al. Room temperature quantum emission from cubic silicon carbide nanoparticles. ACS Nano 8, 7938-7947 (2014).

12. Widmann, M. et al. Coherent control of single spins in silicon carbide at room temperature. Nat. Mater. 14, 164-168 (2015).

13. Christle, D. J. et al. Isolated electron spins in silicon carbide with millisecond coherence times. Nat. Mater. 14, 160-163 (2015).

14. Falk, A. L. et al. Electrically and mechanically tunable electron spins in silicon carbide color centers. Phys. Rev. Lett. 112, 187601 (2014).

15. Fuchs, F. et al. Engineering near-infrared single-photon emitters with optically active spins in ultrapure silicon carbide. Nat. Commun. 6, 7578 (2015).

16. Radulaski, M. et al. Scalable quantum photonics with single color centers in silicon carbide. Nano Lett. 17, 1782-1786 (2017). 
17. Csóré, A., von Bardeleben, H. J., Cantin, J. L. \& Gali, A. Characterization and formation of NV centers in $3 \mathrm{C}, 4 \mathrm{H}$, and $6 \mathrm{H} \mathrm{SiC}$ : an ab initio study. Phys. Rev. B 96, 085204 (2017).

18. von Bardeleben, $\mathrm{H}$. J. et al. $\mathrm{NV}$ centers in $3 \mathrm{C}, 4 \mathrm{H}$, and $6 \mathrm{H}$ silicon carbide: a variable platform for solid-state qubits and nanosensors. Phys. Rev. B 94, 121202 (2016).

19. Lohrmann, A. et al. Single-photon emitting diode in silicon carbide. Nat. Commun. 6, 7783 (2015)

20. Lohrmann, A. et al. Activation and control of visible single defects in $4 \mathrm{H}-, 6 \mathrm{H}-$, and 3C-SiC by oxidation. Appl. Phys. Lett. 108, 021107 (2016).

21. Lienhard, B. et al. Bright and photostable single-photon emitter in silicon carbide. Optica 3, 768 (2016).

22. Lohrmann, A., Johnson, B. C., McCallum, J. C. \& Castelletto, S. A review on single photon sources in silicon carbide. Rep. Prog. Phys. 80, 034502 (2017).

23. Grosso, G. et al. Tunable and high-purity room temperature single-photon emission from atomic defects in hexagonal boron nitride. Nat. Commun. 8, 705 (2017).

24. Chakraborty, C., Kinnischtzke, L., Goodfellow, K. M., Beams, R. \& Vamivakas, A. N. Voltage-controlled quantum light from an atomically thin semiconductor. Nat. Nanotechnol. 10, 507-511 (2015).

25. Saddow, S. E. \& Agarwal, A. K. Advances in Silicon Carbide Processing and Applications. (Artech House, Norwood: Massachusetts, US, 2004).

26. Fuchs, F. et al. Silicon carbide light-emitting diode as a prospective room temperature source for single photons. Sci. Rep. 3, 1637 (2013).

27. Boretti, A., Rosa, L., Mackie, A. \& Castelletto, S. Electrically driven quantum light sources. Adv. Opt. Mater. 3, 1012-1033 (2015).

28. Mizuochi, N. et al. Electrically driven single-photon source at room temperature in diamond. Nat. Photon. 6, 299-303 (2012).

29. Lohrmann, A. et al. Diamond based light-emitting diode for visible single-photon emission at room temperature. Appl. Phys. Lett. 99, 251106 (2011).

30. Berhane, A. M. et al. Electrical excitation of silicon-vacancy centers in single crystal diamond. Appl. Phys. Lett. 106, 171102 (2015).

31. Choi, S. et al. Electroluminescence from localized defects in zinc oxide: toward electrically driven single photon sources at room temperature. ACS Appl. Mater. Interfaces 7, 5619-5623 (2015).

32. Deshpande, S., Frost, T., Hazari, A. \& Bhattacharya, P. Electrically pumped singlephoton emission at room temperature from a single InGaN/GaN quantum dot. Appl. Phys. Lett. 105, 141109 (2014).

33. Aharonovich, I., Englund, D. \& Toth, M. Solid-state single-photon emitters. Nat. Photon. 10, 631-641 (2016).

34. Eberlein, T. A. G., Jones, R., Öberg, S. \& Briddon, P. R. Density functional theory calculation of the $D_{1}$ optical center in SiC. Phys. Rev. 74, 144106 (2006).

35. Brillson, L. J., Tumakha, S., Okojie, R. S., Zhang, M. \& Pirouz, P. Electron-excited luminescence of SiC surfaces and interfaces. J. Phys. Condens. Matter 16, S1733-S1754 (2004)

36. Gali, A. et al. Correlation between the antisite pair and the $D_{1}$ center in SiC. Phys. Rev. B 67, 155203 (2003)

37. Fedyanin, D. Yu. \& Agio, M. Ultrabright single-photon source on diamond with electrical pumping at room and high temperatures. New J. Phys. 18, 073012 (2016).

38. Torpo, L., Marlo, M., Staab, T. E. M. \& Nieminen, R. M. Comprehensive ab initio study of properties of monovacancies and antisites in $4 \mathrm{H}-\mathrm{SiC}$. J. Phys. Condens. Matter 13, 6203-6231 (2001).
39. Friedrichs, P., Kimoto, T., Ley, L. \& Pensl, G. Silicon Carbide: Volume 1: Growth, Defects, and Novel Applications. (Wiley-VCH: Weinheim, Germany, 2011).

40. Claeys, C. \& Simoen, E. Radiation Effects in Advanced Semiconductor Materials and Devices. (Springer: Berlin, Germany, 2002).

41. Abakumov, V. N, Perel, V. I. \& Yassievich, I. N. Nonradiative Recombination in Semiconductors. (Elsevier: Amsterdam, Netherlands, 1991).

42. Abakumov, V. N. \& lassievich, I. N. Cross section for the recombination of an electron on a positively charged center in a semiconductor. JETP Lett. 71, 657-664 (1976).

43. Smirnov, B. M. Fundamentals of lonized Gases. (Wiley-VCH: Weinheim, Germany, 2011).

44. Khramtsov, I. A., Agio, M. \& Fedyanin, D. Yu. Dynamics of single-photon emission from electrically pumped color centers. Phys. Rev. Appl. 8, 024031 (2017).

45. Vyshnevyy, A. A. \& Fedyanin, D. Yu. Self-heating and cooling of active plasmonic waveguides. ACS Photonics 3, 51-57 (2016)

46. Agio, M. \& Alù, A. Optical Antennas. (Cambridge Univ. Press: Cambridge, UK, 2013).

47. Koenderink, A. F. Single-photon nanoantennas. ACS Photonics 4, 710-722 (2017).

48. Kinkhabwala, A. et al. Large single-molecule fluorescence enhancements produced by a bowtie nanoantenna. Nat. Photon. 3, 654-657 (2009).

49. Eggleston, M. S., Messer, K., Zhang, L., Yablonovitch, E. \& Wu, M. C. Optical antenna enhanced spontaneous emission. Proc. Natl Acad. Sci. USA 112, 1704-1709 (2015)

50. Akselrod, G. M. et al. Probing the mechanisms of large Purcell enhancement in plasmonic nanoantennas. Nat. Photon. 8, 835-840 (2014).

51. Evwaraye, A. O., Smith, S. R. \& Mitchel, W. C. Shallow and deep levels in $\mathrm{n}$-type 4H-SiC. J. Appl. Phys. 79, 7726-7730 (1996).

52. Negoro, Y., Kimoto, T., Matsunami, H., Schmid, F. \& Pensl, G. Electrical activation of high-concentration aluminum implanted in 4H-SiC. J. Appl. Phys. 96, 4916-4922 (2004).

53. Laube, $\mathrm{M}$. et al. Electrical activation of high concentrations of $\mathrm{N}$ and $\mathrm{P}$ ions implanted into 4H-SiC. J. Appl. Phys. 92, 549-554 (2002).

54. Esteban, R., Teperik, T. V. \& Greffet, J. J. Optical patch antennas for single photon emission using surface plasmon resonances. Phys. Rev. Lett. 104, 026802 (2010).

55. Stenger, I. et al. Impurity-to-band activation energy in phosphorus doped diamond. J. Appl. Phys. 114, 073711 (2013).

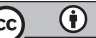

Open Access This article is licensed under a Creative Commons Attribution 4.0 International License, which permits use, sharing, adaptation, distribution and reproduction in any medium or format, as long as you give appropriate credit to the original author(s) and the source, provide a link to the Creative Commons license, and indicate if changes were made. The images or other third party material in this article are included in the article's Creative Commons license, unless indicated otherwise in a credit line to the material. If material is not included in the article's Creative Commons license and your intended use is not permitted by statutory regulation or exceeds the permitted use, you will need to obtain permission directly from the copyright holder. To view a copy of this license, visit http://creativecommons. org/licenses/by/4.0/.

(c) The Author(s) 2018 